\title{
Formaldehyde Oxidation over Co@N-Doped Carbon at Room Temperature: Tunable Co Size and Intensified Surface Electron Density
}

Dandan Zhu ${ }^{a, b}$, Yu Huang ${ }^{b, c, *}$, Rong Li $i^{b, c}$, Tingting Huang ${ }^{b, c}$, Jun-ji Cao ${ }^{b, c}$, Zhenxing Shen $^{a, b, *}$, Shun Cheng Lee ${ }^{d}$

${ }^{\text {a }}$ Department of Environmental Sciences and Engineering, Xi'an Jiaotong University, Xi'an 710049, China

${ }^{\mathrm{b}}$ Key Lab of Aerosol Chemistry \& Physics, SKLLQG, Institute of Earth Environment, Chinese Academy of Sciences, Xi' an 710061, China

${ }^{\mathrm{c}}$ CAS Center for Excellence in Quaternary Science and Global Change, Xi' an 710061, China

${ }^{\mathrm{d}}$ Department of Civil and Environmental Engineering, The Hong Kong Polytechnic University, Hong Kong, China

*Corresponding author, E-mail address: huangyu@ieecas.cn (Yu Huang)

The Supporting Information contains 23 pages, including Section S1, S2, and S3, Scheme S1, and Figures S1-S17. 


\section{Section S1: Materials and Precursors Preparation}

Cobalt nitrate hexahydrate $\left(\mathrm{Co}\left(\mathrm{NO}_{3}\right)_{2} \cdot 6 \mathrm{H}_{2} \mathrm{O}\right)$ and Zinc nitrate hexahydrate $\left(\mathrm{Zn}\left(\mathrm{NO}_{3}\right)_{2} \cdot 6 \mathrm{H}_{2} \mathrm{O}\right)$ were purchased from Sinopharm Chemical Reagent Co., Ltd. 2-methylimidazole (2-mIm) was bought from Aladdin Industrial Corporation. Absolute methanol and ethanol with analytical grade were provided by Tianjin Hengxing Chemical Reagent Co., Ltd. All chemicals were used as received without further purification. Deionized water $(18.2 \mathrm{M} \Omega \cdot \mathrm{cm})$ was obtained by a Millipore system.

Co-ZIF precursors were synthesized according to the previously reported method ${ }^{1}$. For precursor with pure $\mathrm{Co}^{2+}$ as metal ions (i.e., ZIF-67), $2.935 \mathrm{~g}$ of $\mathrm{Co}\left(\mathrm{NO}_{3}\right)_{2} \cdot 6 \mathrm{H}_{2} \mathrm{O}$ and $6.5 \mathrm{~g}$ of 2-mIm were dissolved in 80 and $40 \mathrm{~mL}$ methanol, respectively, followed by mixing these two methanolic solutions rapidly, and stirring for $24 \mathrm{~h}$ at ambient temperature. The dark purple product denoted as Co-ZIF-1.0 was obtained through centrifugation, washing with ethanol, and drying in a vacuum oven at $80^{\circ} \mathrm{C}$ overnight. The precursors with different cobalt contents were prepared through the identical procedure, except for simultaneous dissolution of $\mathrm{Co}\left(\mathrm{NO}_{3}\right)_{2} \cdot 6 \mathrm{H}_{2} \mathrm{O}$ and $\mathrm{Zn}\left(\mathrm{NO}_{3}\right)_{2} \cdot 6 \mathrm{H}_{2} \mathrm{O}$ with total amounts of $10 \mathrm{mmol}$. The resultant precursors were referred as Co-ZIF- $x(x=0.8,0.5,0.25,0.2,0.05,0)$, where $x$ represents the $\mathrm{Co}^{2+}$ molar ratio against total metal ions. 


\section{Section S2: Physicochemical Characterizations}

Scanning electron microscopy (SEM) analyses were conducted on a MAIA3 microscope (TESCAN, Czech) equipped with energy disperse spectroscopy (EDS, Bruker, German) at an acceleration voltage of $20 \mathrm{kV}$. Nitrogen adsorption-desorption isotherms were measured on a surface area and porosimetry analyzer (ASAP 2460, Micromeritics Instrument Corp., USA) at $77 \mathrm{~K}$, with all samples evacuated at $150{ }^{\circ} \mathrm{C}$ for $12 \mathrm{~h}$ under high vacuum before measurements. 


\section{Section S3: Computational Methods}

All calculations have been carried out using the periodic spin-polarized DFT implemented in the Vienna Ab Initio Simulation Package (VASP) ${ }^{2-3}$. The generalized gradient approximation with Perdew-Burke-Ernzerhof exchange-correlation functional (GGA-PBE) is used $^{4}$. The kinetic energy cutoff for a plane wave basis set is $400 \mathrm{eV}$, a Monkhorst-Pack mesh k-points of $2 \times 2 \times 2$ is used ${ }^{5}$, and the projector augmented wave pseudopotentials are used to describe the inner-shell electrons. When the change of total energy is converged to $5 \times 10^{-6} \mathrm{eV} /$ atom, and the forces on the ions are below $10^{-2} \mathrm{eV} / \AA$, the optimization is thought to be converged. 


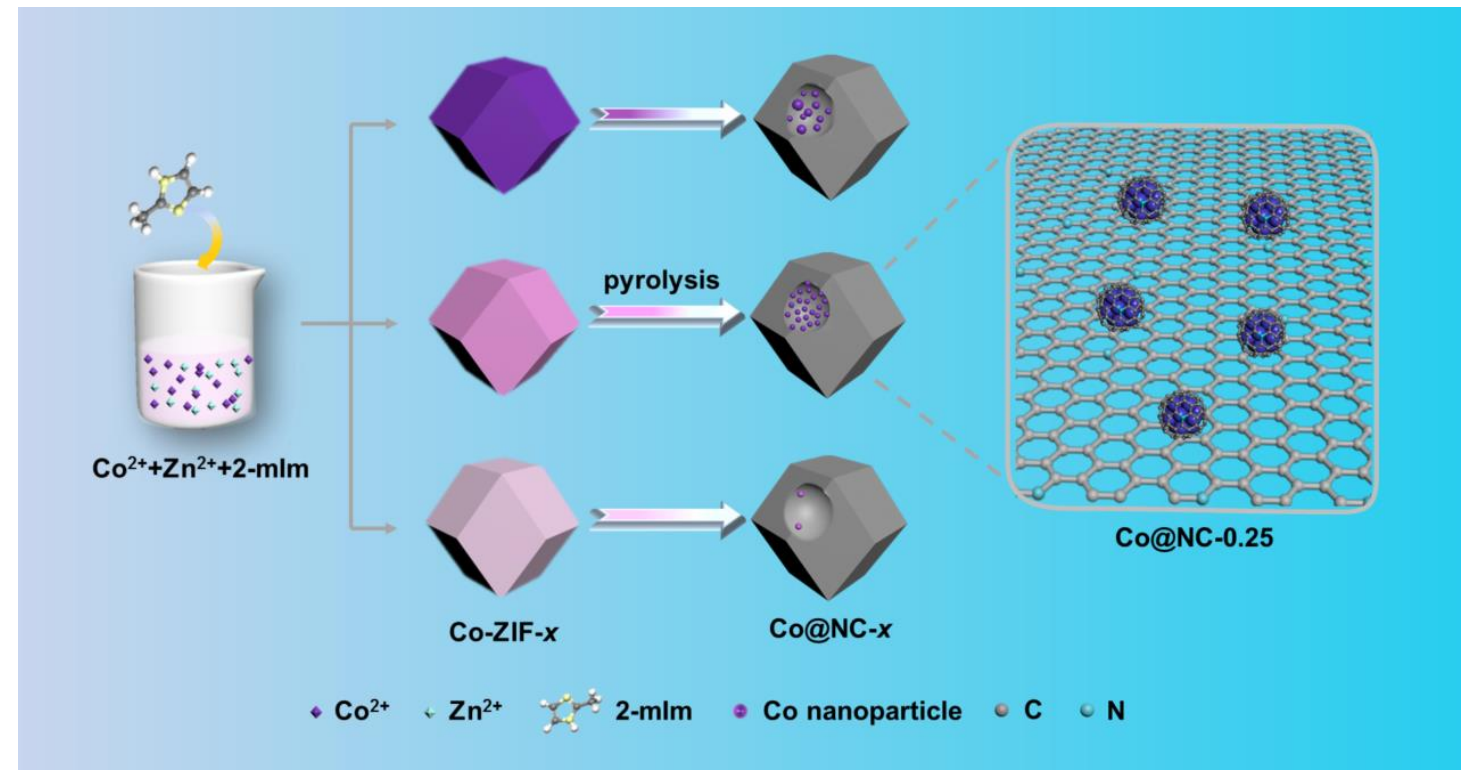

Scheme S1. Schematic illustration for the preparation of Co@NC- $x(x=0.5,0.25,0.05$ from top to bottom) series nanocomposites. 

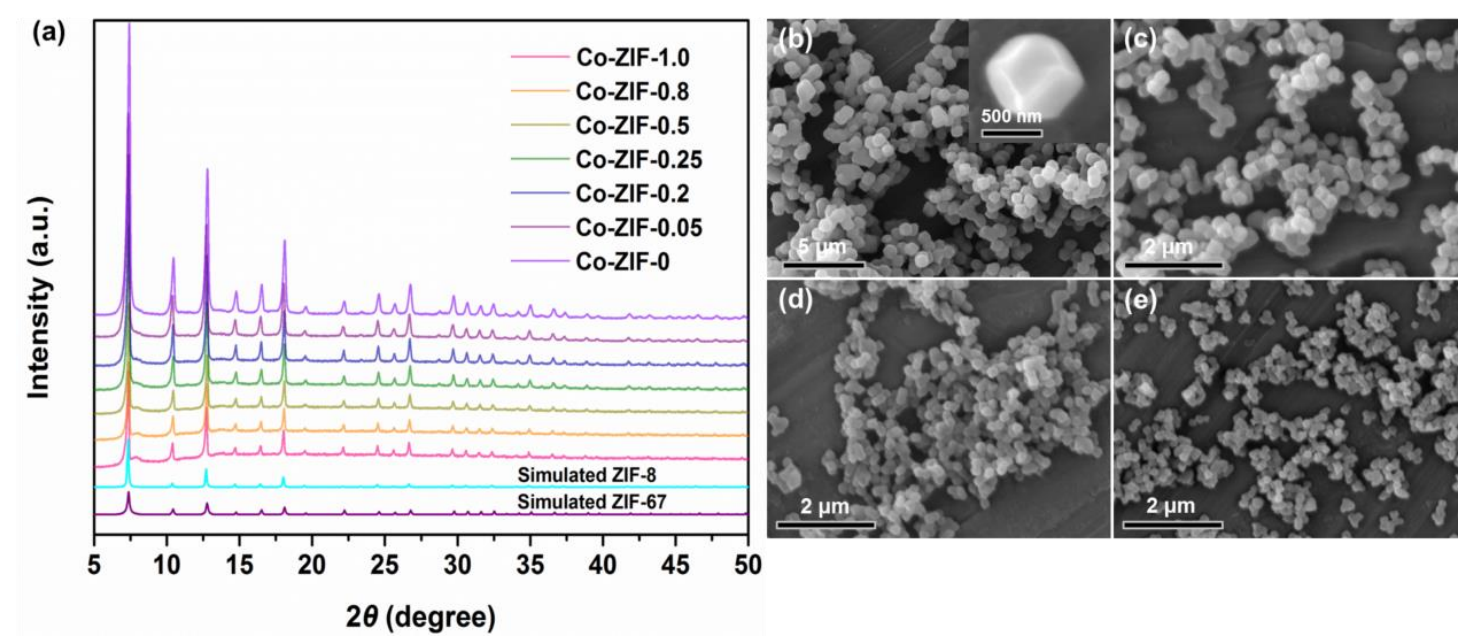

Figure S1. (a) Theoretical XRD spectra of ZIF-67 and ZIF-8, and experimental XRD patterns of Co-ZIF- $x(x=1.0,0.8,0.5,0.25,0.2,0.05,0)$. (b-e) SEM images of Co-ZIF-1.0, Co-ZIF0.5, Co-ZIF-0.25, and Co-ZIF-0.05.

ZIF precursors with different adding amounts of cobalt ions were examined by XRD. Figure S1a displayed that the diffraction peaks of all precursors matched well with those of simulated ZIF-8 and ZIF-67, indicating the as-prepared precursors possessed the high purity and crystallinity. The SEM images in Figure S1b shows Co-ZIF-1.0 possesses the dodecahedron with about $700 \mathrm{~nm}$, and other precursors also display the similar morphologies. The decreased size was caused by the elevated nucleation and lowered growth rate of ZIF crystal, since the adding $\mathrm{Zn}^{2+}$ could promote nucleation of crystal core. 

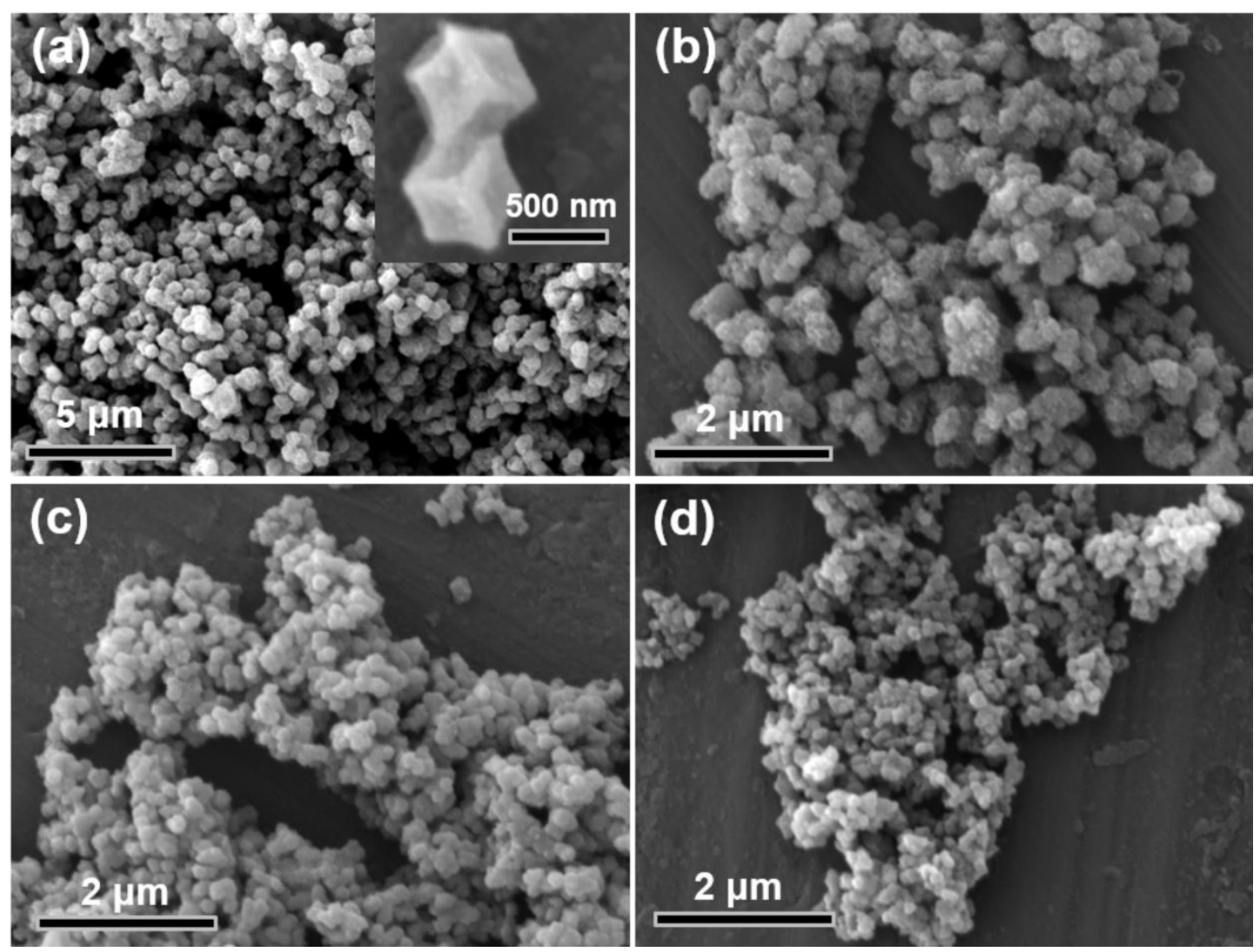

Figure S2. SEM images of Co@NC-1.0 (a), Co@NC-0.5 (b), Co@NC-0.25 (c), and $\mathrm{Co} @ \mathrm{NC}-0.05$ (d). 

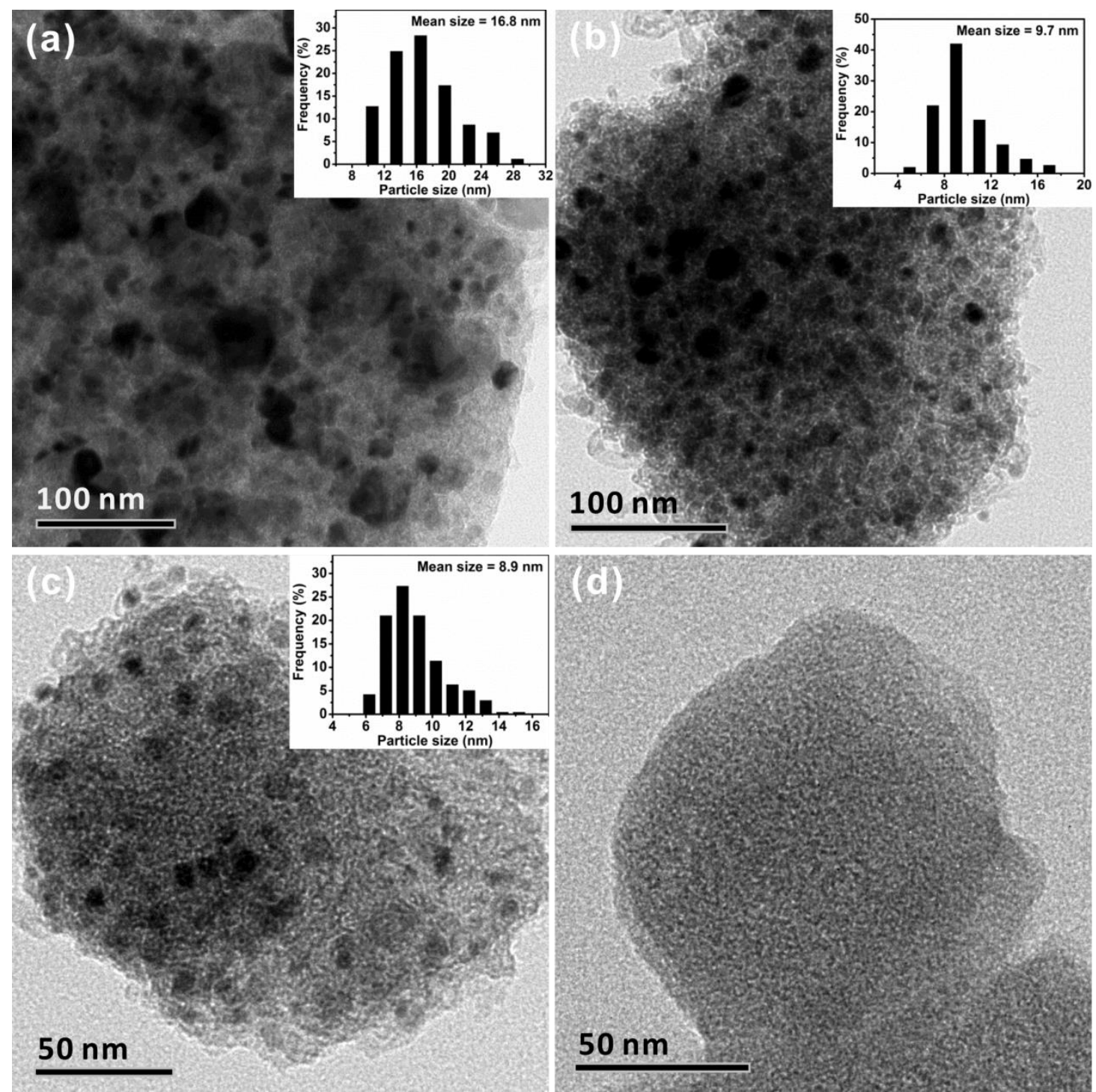

Figure S3. TEM images of $\mathrm{Co} @ \mathrm{NC}-1.0$ (a), Co@NC-0.5 (b), Co@NC-0.25 (c), and

Co@NC-0.05 (d). Insets in panel (a-c) are the size distributions of Co NPs in Co@NC-1.0,

Co@NC-0.5, and Co@NC-0.25. 


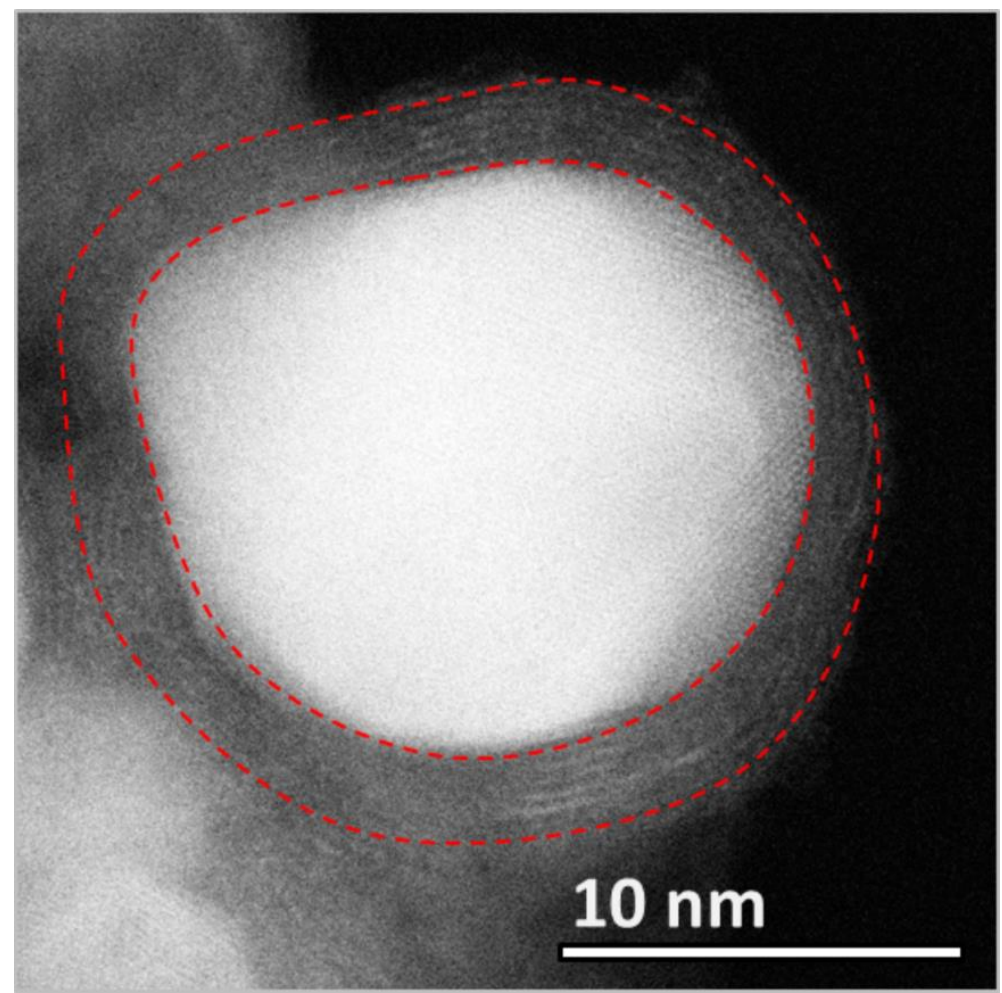

Figure S4. HAADF-STEM image of Co@NC-0.25. 


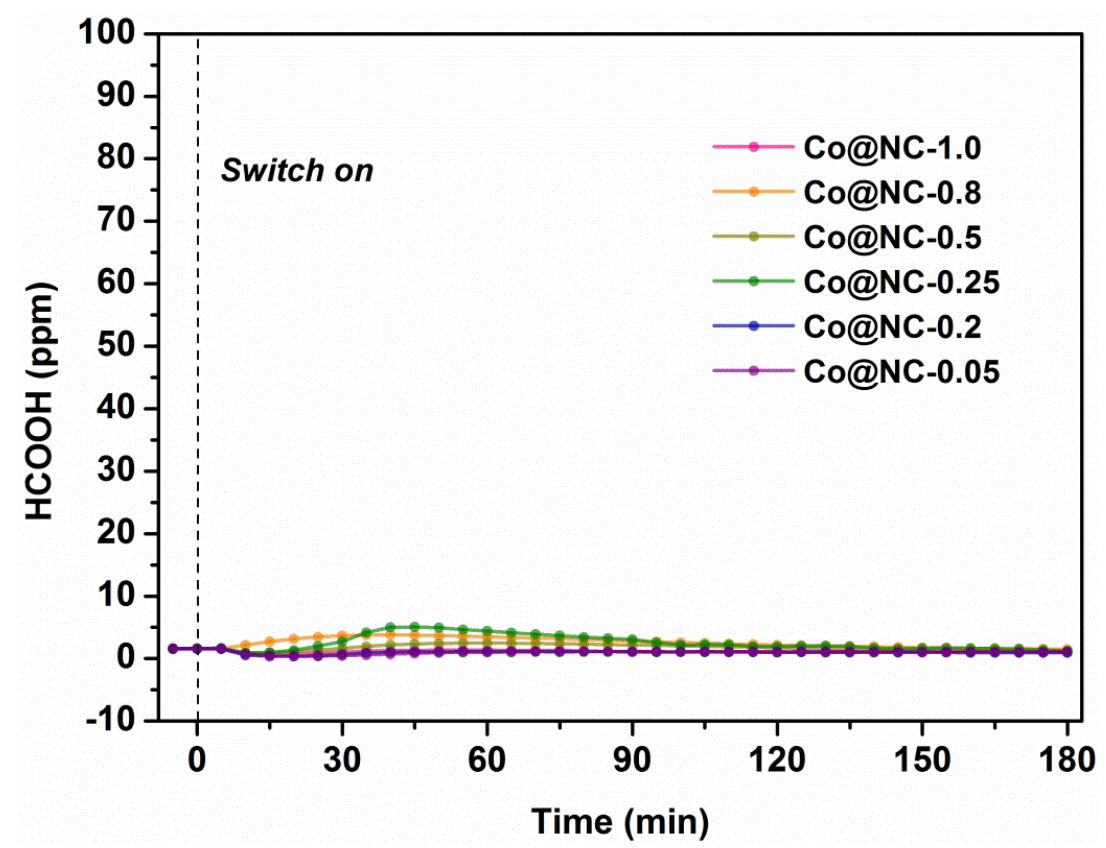

Figure S5. The concentration of $\mathrm{HCOOH}$ as a function of time during $\mathrm{HCHO}$ abatement over Co@NC-x $(x=1.0,0.8,0.5,0.25,0.2,0.05)$. 

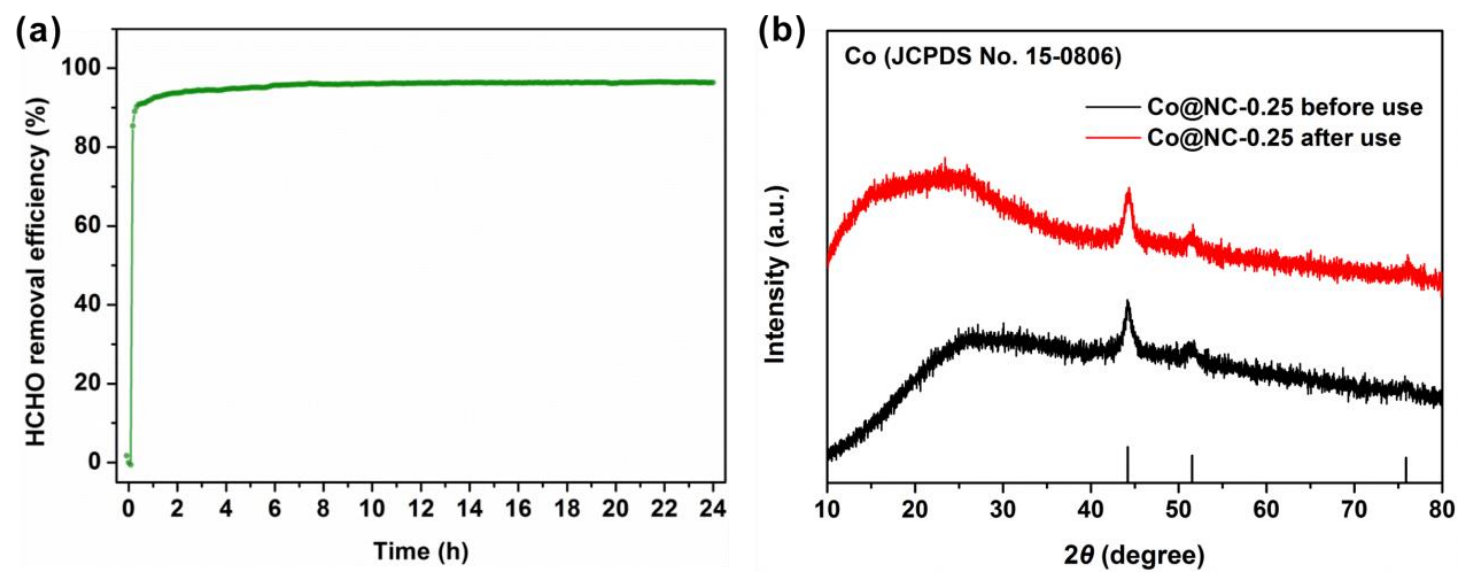

Figure S6. (a) Durability testing of Co@NC-0.25 for HCHO removal; (b) XRD patterns of Co@NC- 0.25 before and after HCHO oxidation reaction for $24 \mathrm{~h}$. Reaction conditions:

HCHO 100 ppm in air; total flow rate $100 \mathrm{~mL} \cdot \mathrm{min}^{-1}$; RH $25 \%$. 

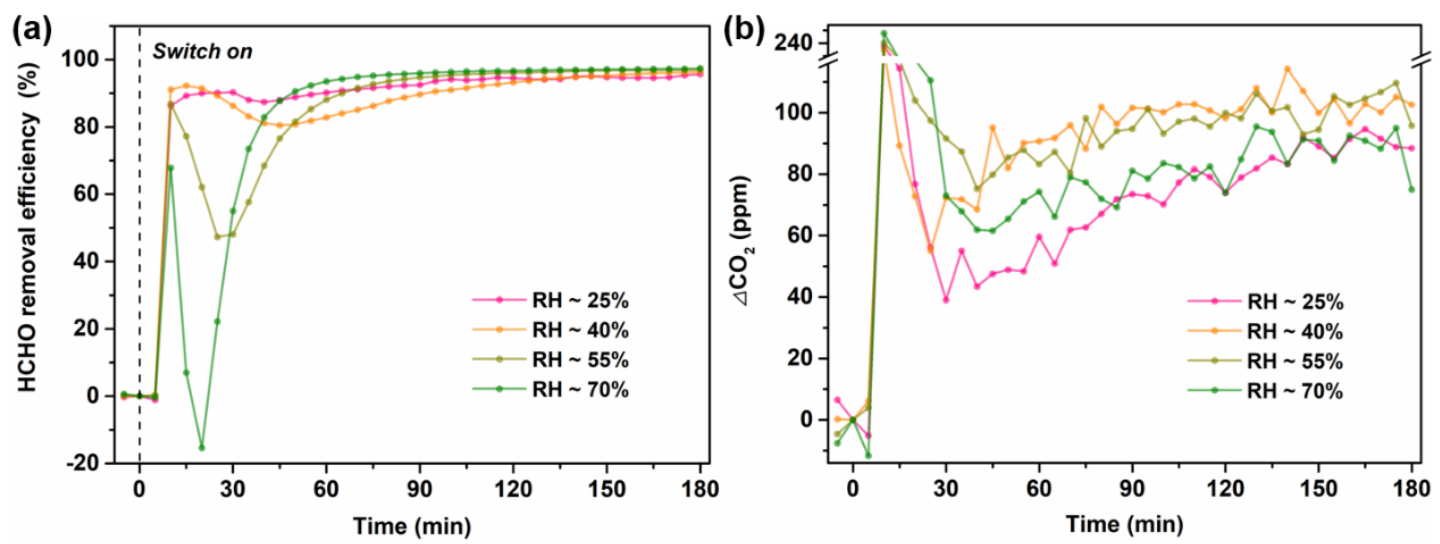

Figure S7. (a) HCHO removal efficiency and (b) the generated amounts of $\mathrm{CO}_{2}$ as a function of time under different RH over Co@NC-0.25. 

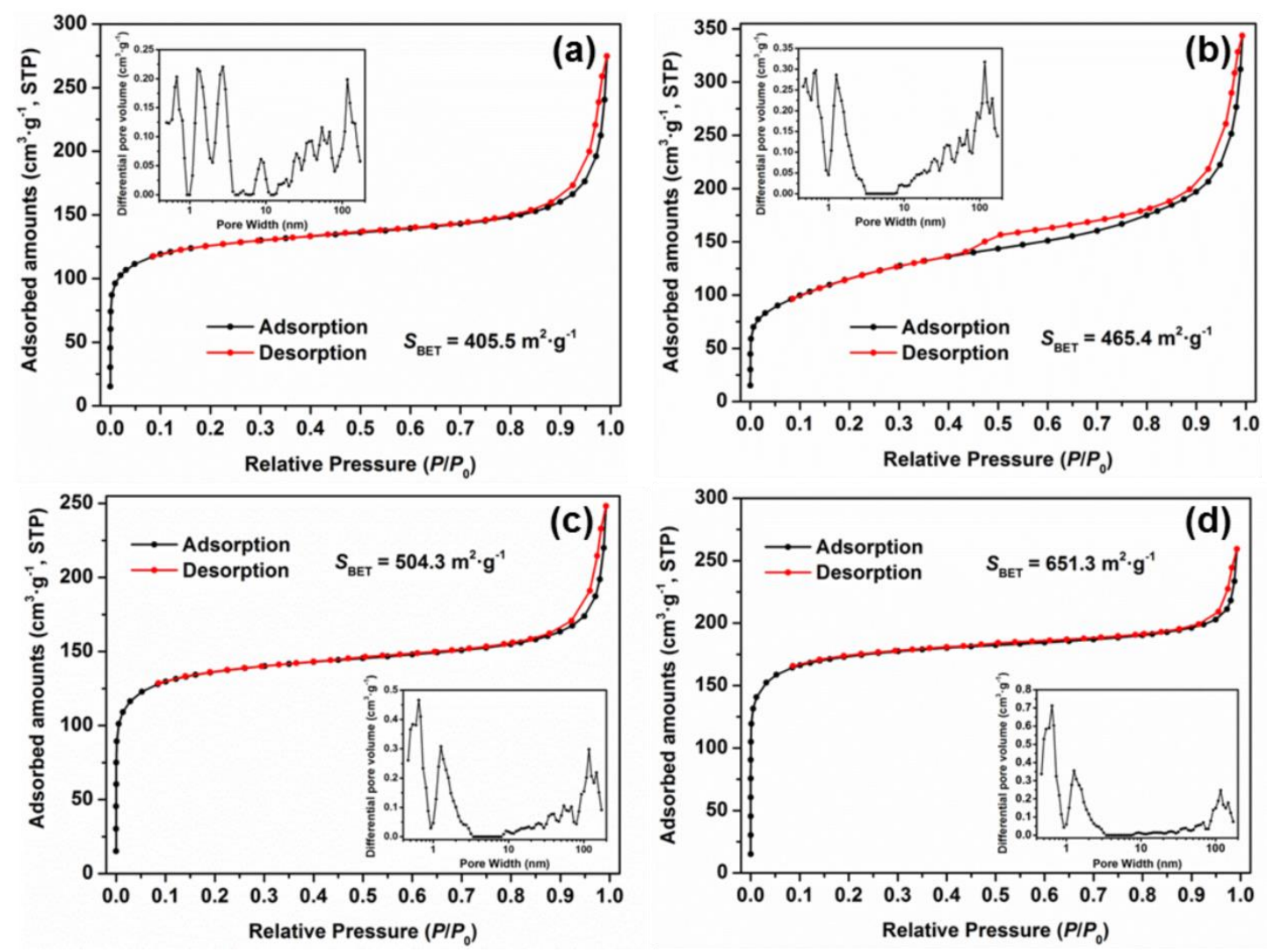

Figure S8. $\mathrm{N}_{2}$ adsorption-desorption isotherms of Co@NC-0.5 (a), Co@NC-0.25 (b),

Co@NC-0.2 (c), and Co@NC-0.05 (d). Insets are pore-width distribution curves of the corresponding catalysts $(\mathrm{STP}=$ standard temperature and pressure). 


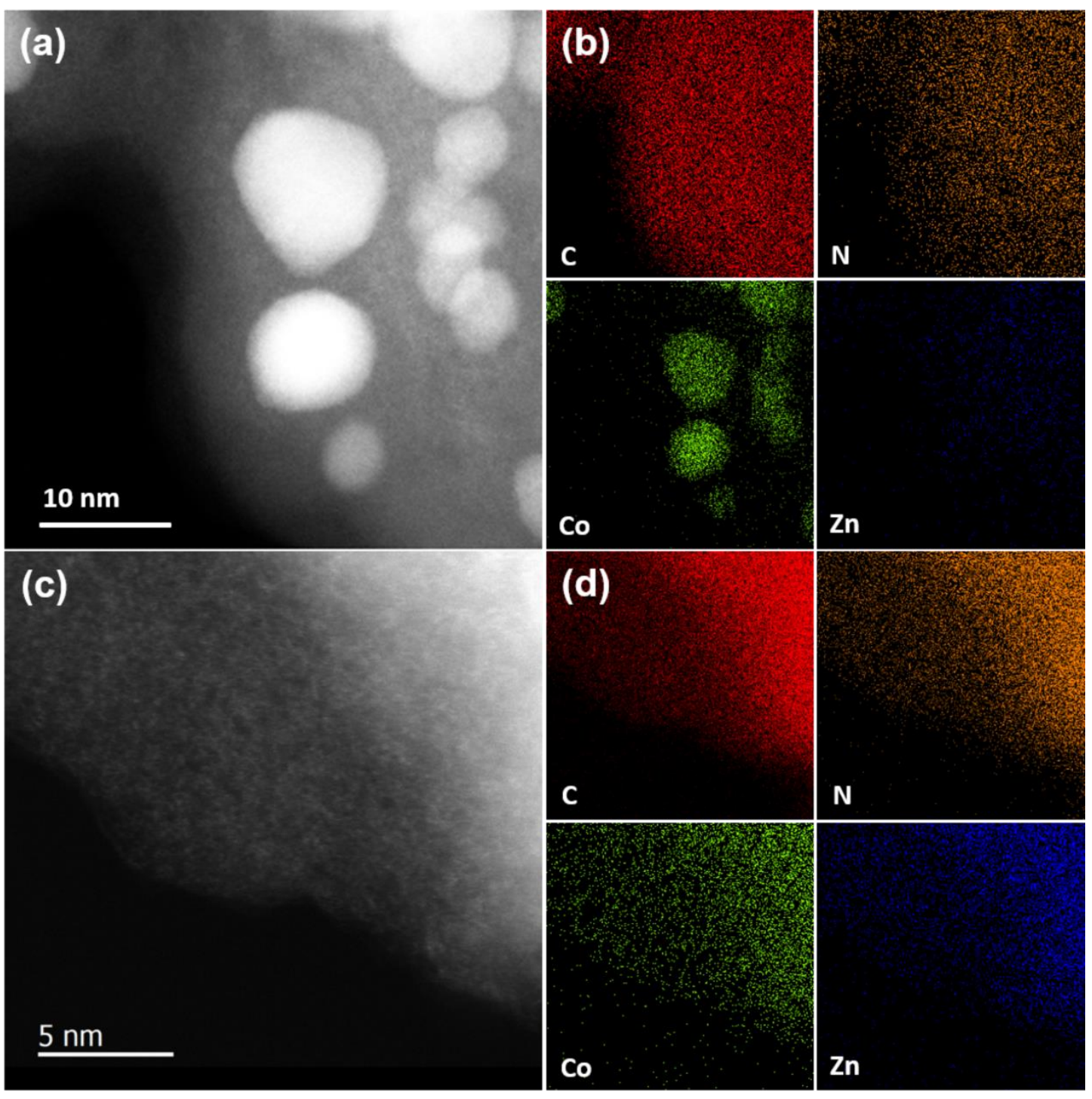

Figure S9. HAADF-STEM images and the corresponding elemental mappings of C, N, Co, and Zn elements for Co@NC-0.25 at two different zones with $(\mathrm{a}, \mathrm{b})$ or without $(\mathrm{c}, \mathrm{d})$ metallic Co NPs. 


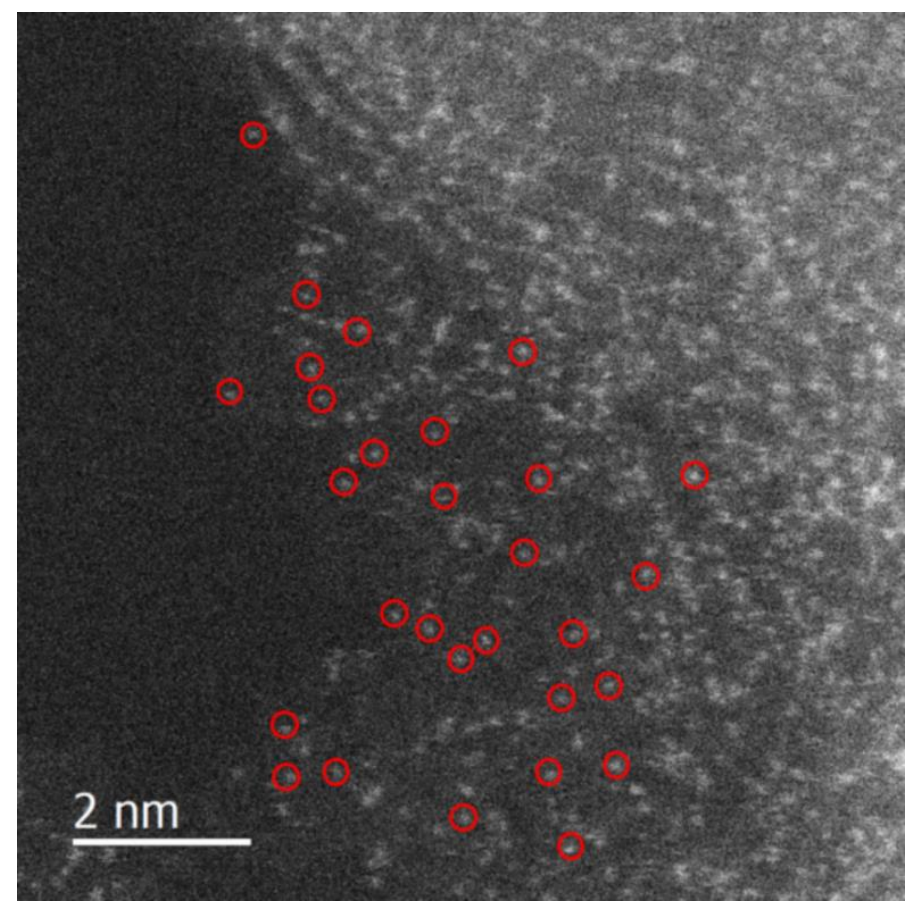

Figure S10. Atomic-resolution spherical aberration-corrected STEM (AC-STEM) image of Co@NC-0.25. 


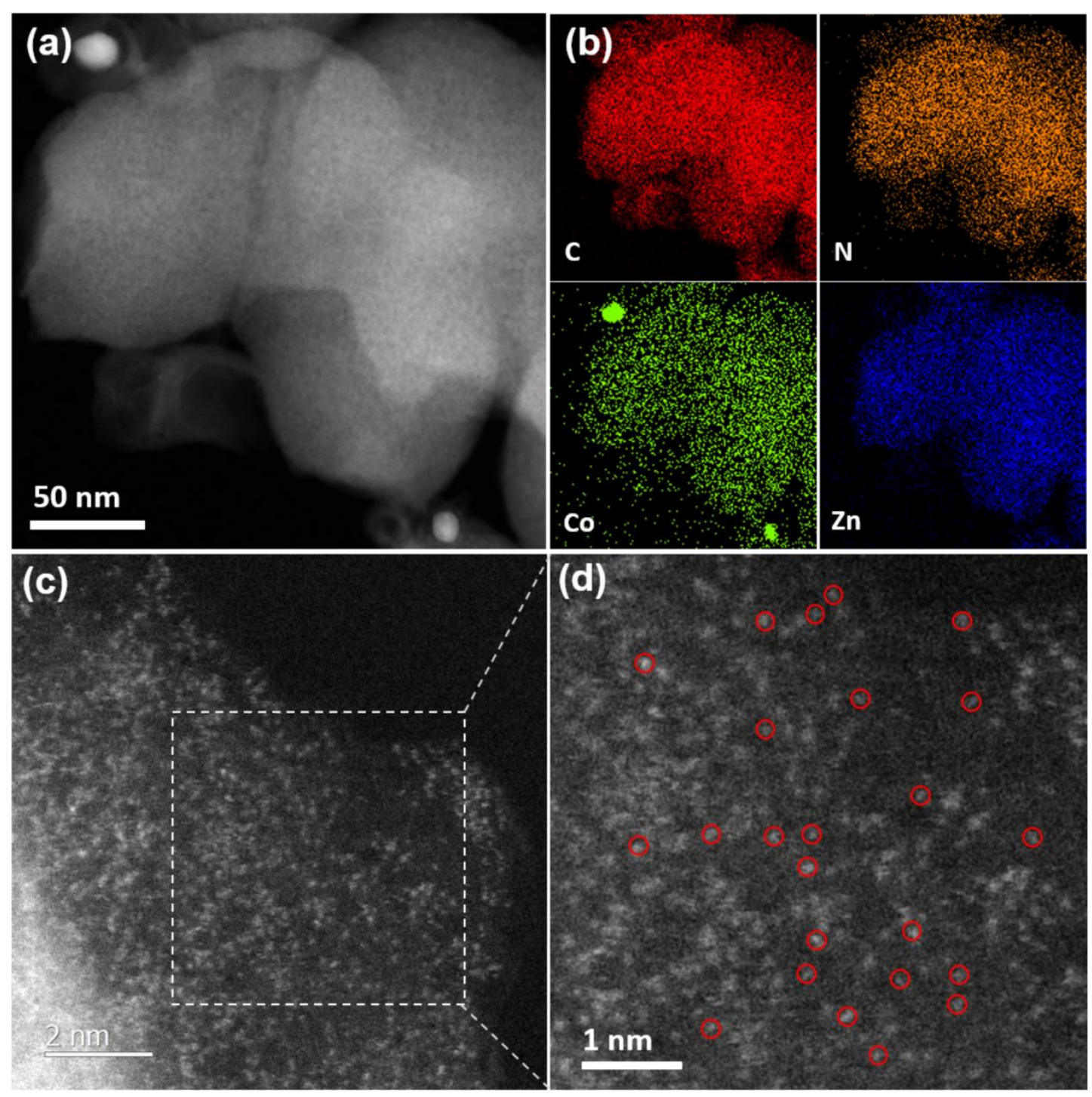

Figure S11. (a, b) HAADF-STEM image and the corresponding elemental mappings of C, N, Co, and Zn elements for Co@NC-0.05; (c, d) AC-STEM image and the enlarged one of Co@NC-0.05. 

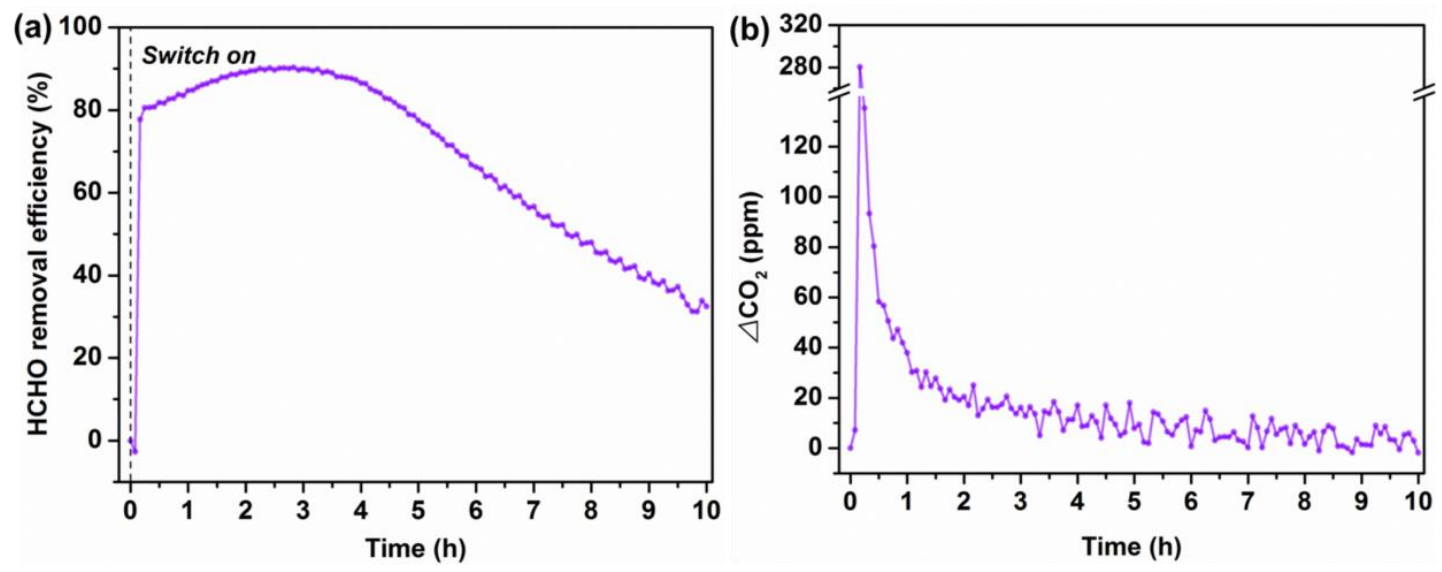

Figure S12. (a) HCHO removal efficiency and (b) the generated amounts of $\mathrm{CO}_{2}$ as a function of time over Co@NC-0 for 10 h. 

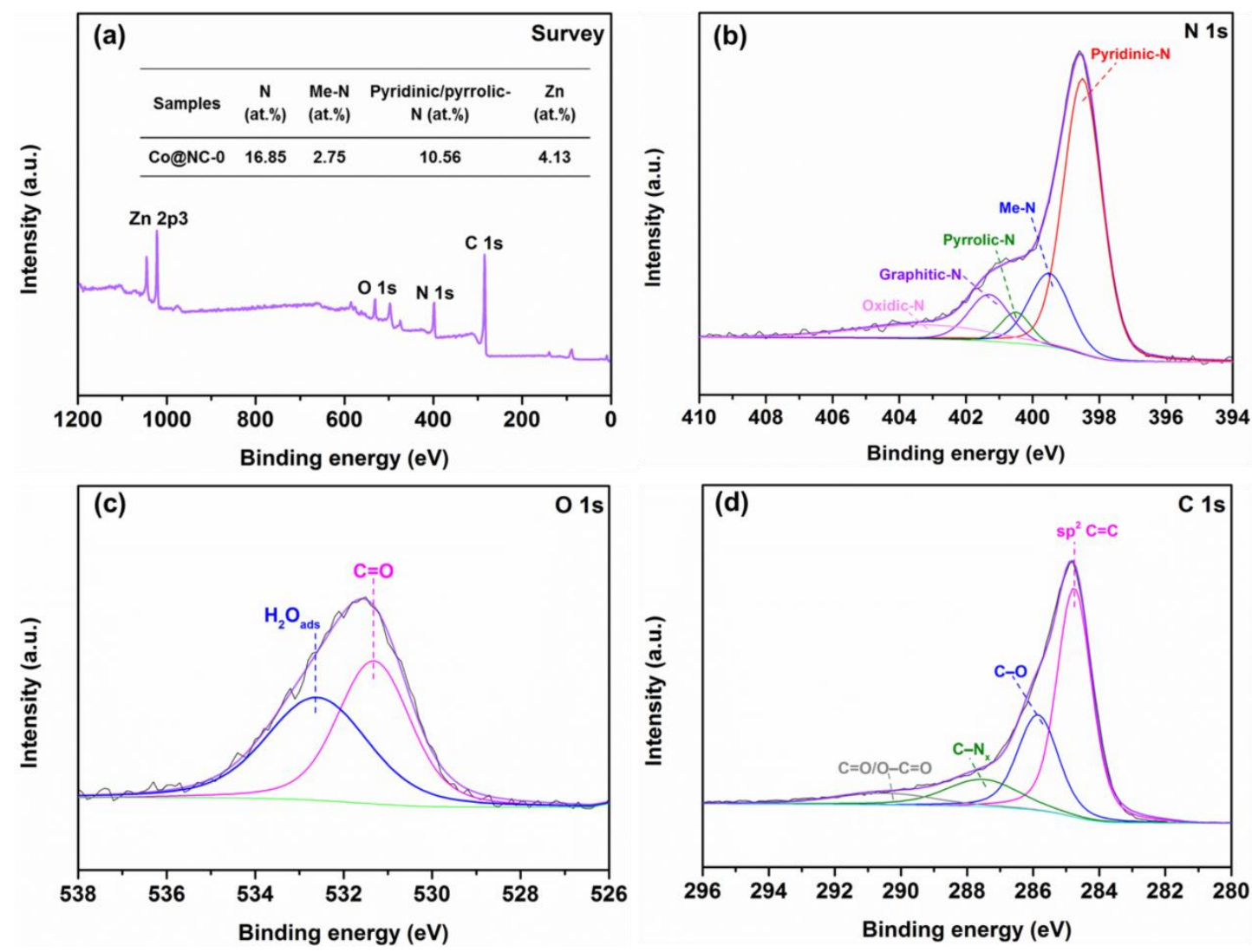

Figure S13. (a) XPS survey spectrum of Co@NC-0; high-resolution N 2p (b), O 1s (c), and C 1s (d) spectra of Co@NC-0. Inset in panel (a) shows the element contents in Co@NC-0 obtained from XPS. 
(a)
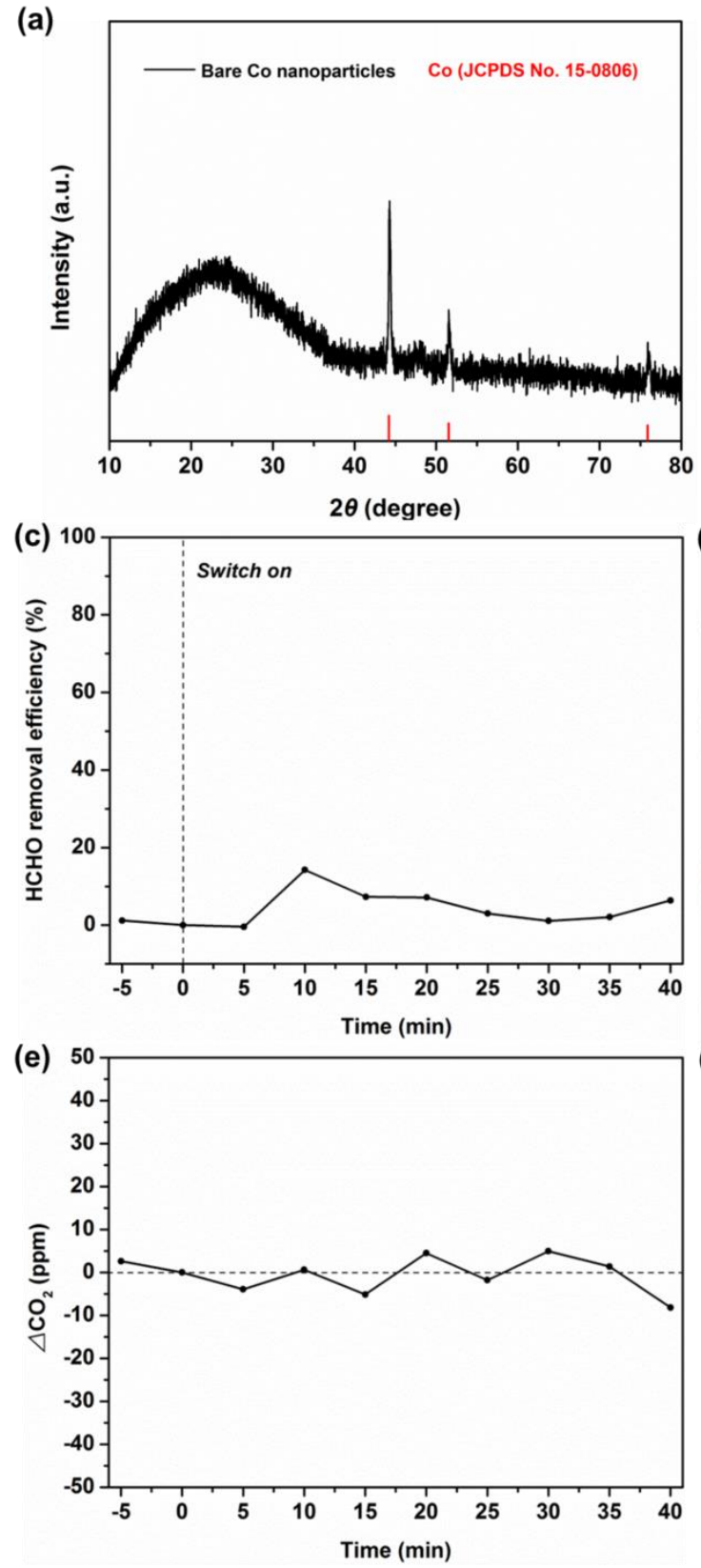
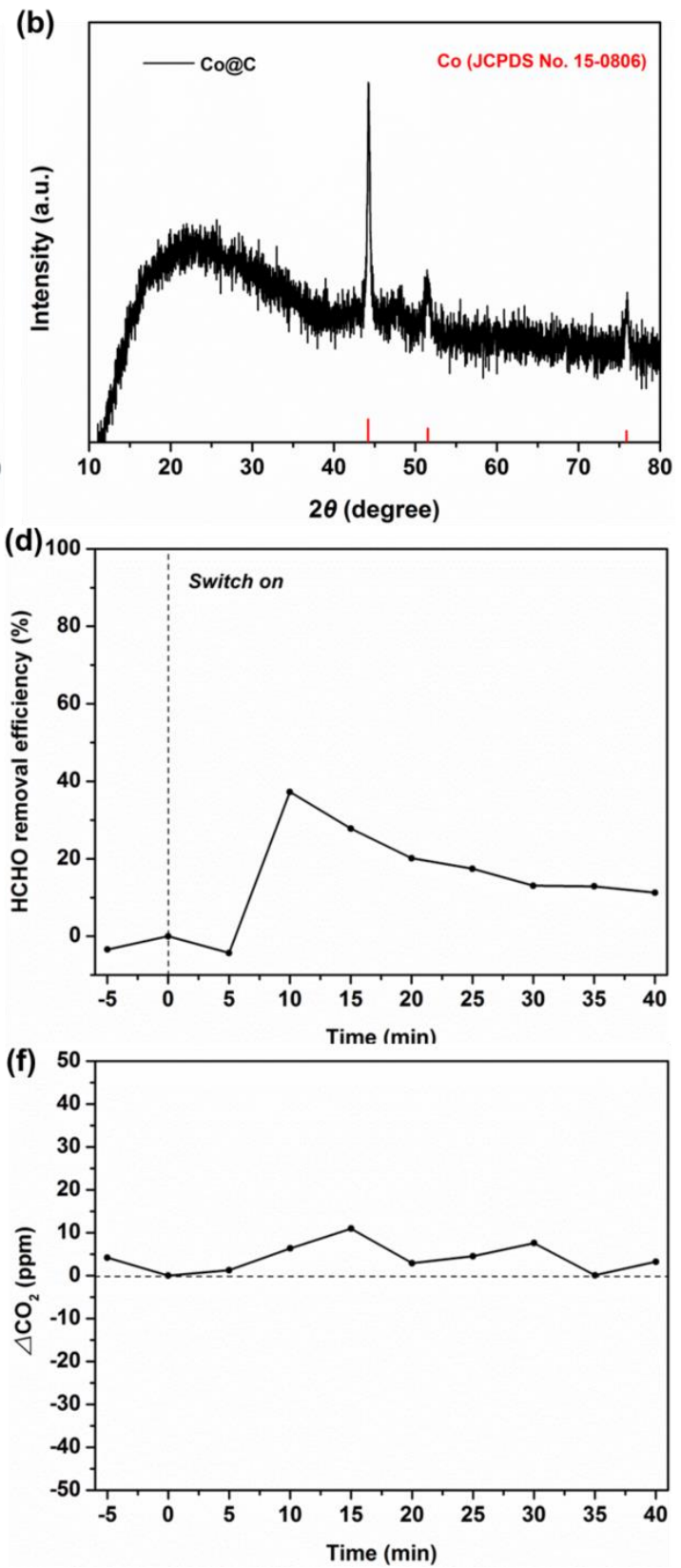

Figure S14. XRD spectra of (a) bare metallic Co particles prepared by calcining cobalt nitrate hexahydrate followed by reduction with $\mathrm{H}_{2}$ and (b) $\mathrm{Co} @ \mathrm{C}$ derived from Co-MOF without nitrogen-containing ligands. $\mathrm{HCHO}$ removal efficiency and the concentration of $\mathrm{CO}_{2}$ generated as a function of time for bare metallic Co particles (c, e) and Co@C (d, f). 
(a)

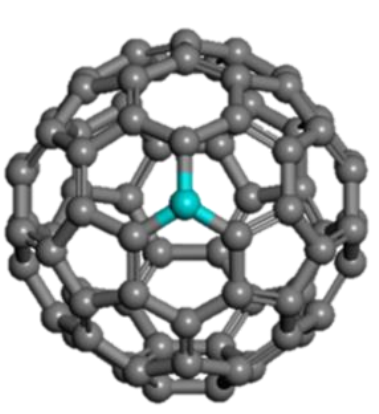

(c)

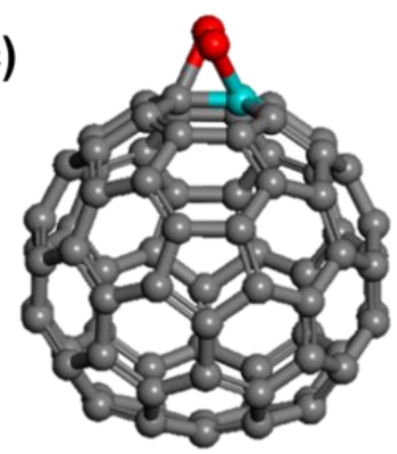

(b)

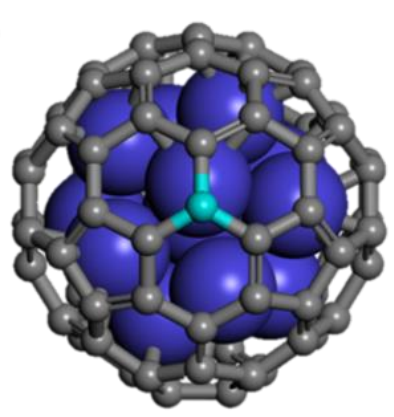

(d)

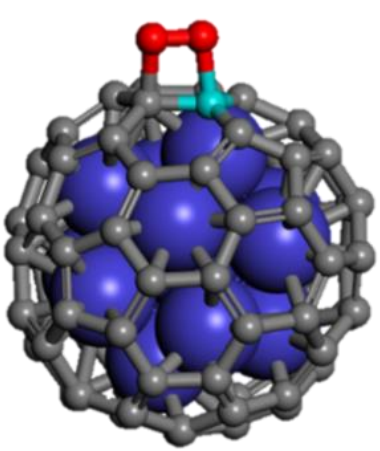

Figure S15. Theoretical models of $\mathrm{C}_{75} \mathrm{~N}_{1}$ (a) and $\mathrm{C}_{75} \mathrm{~N}_{1} \mathrm{Co}_{13}(\mathrm{~b})$, and the corresponding $\mathrm{O}_{2}$ adsorbed structures on $\mathrm{C}_{75} \mathrm{~N}_{1}$ (c) and $\mathrm{C}_{75} \mathrm{~N}_{1} \mathrm{Co}_{13}(\mathrm{~d})$ before optimization. The red, grey, blue, and light blue balls represent $\mathrm{O}, \mathrm{C}, \mathrm{Co}$, and $\mathrm{N}$ atoms, respectively. 


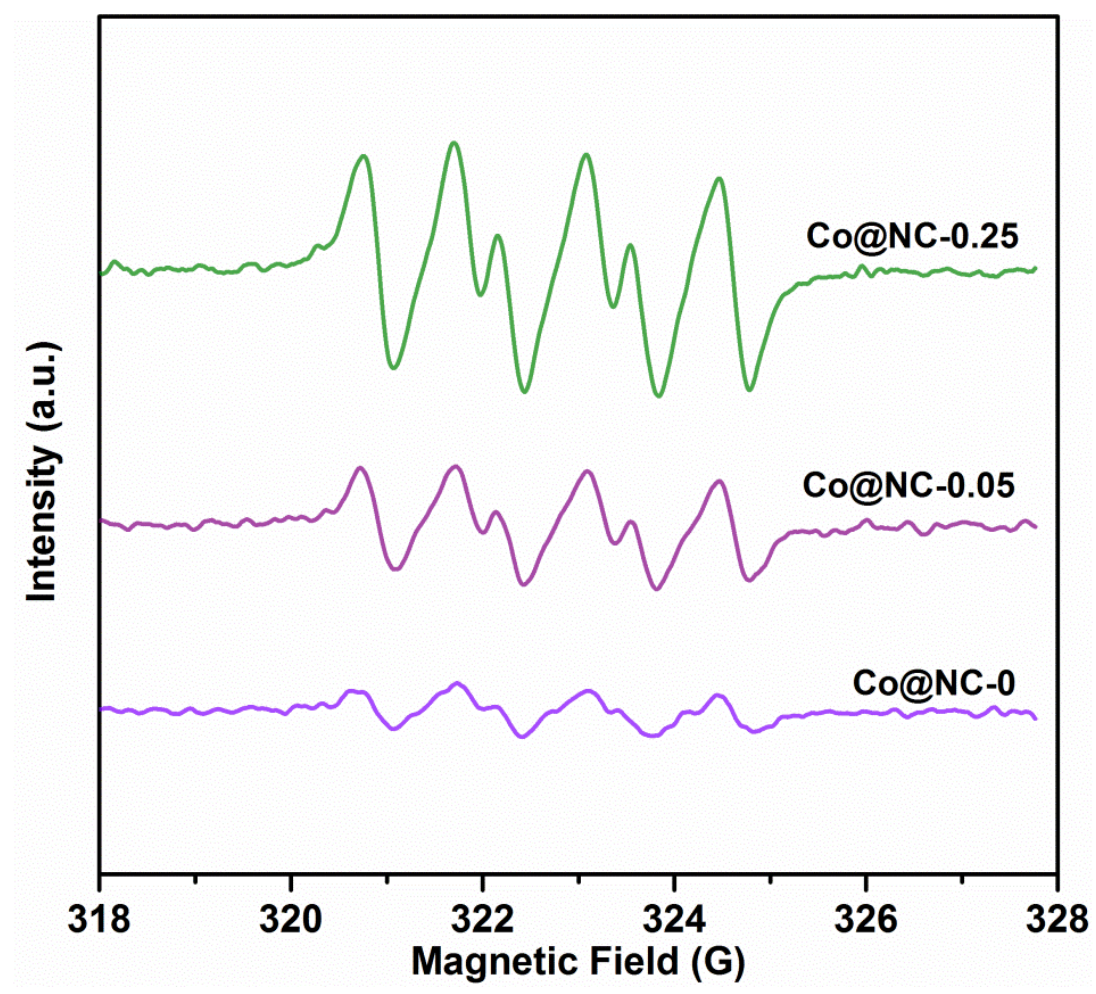

Figure S16. DMPO-trapped EPR spectra of $\cdot \mathrm{O}_{2}{ }^{-}$measured at room temperature under dark for Co@NC-0.25, Co@NC-0.05, and Co@NC-0. 

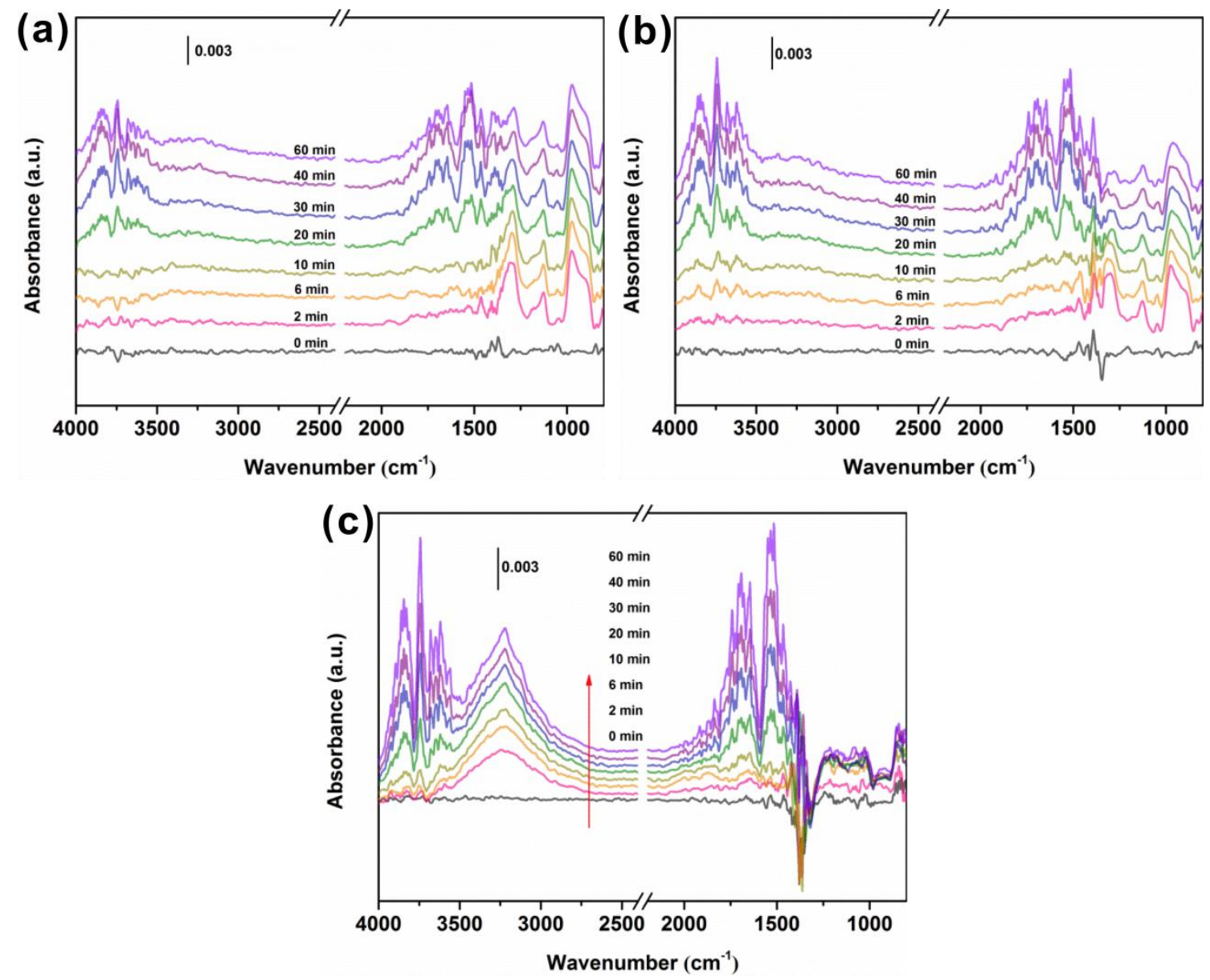

Figure S17. In situ DRIFT spectra of $\mathrm{O}_{2}+\mathrm{HCHO}+\mathrm{H}_{2} \mathrm{O}+\mathrm{N}_{2}$ gas mixture exposure for up to 60 min at room temperature over Co@NC-1.0 (a) and Co@NC-0.5 (b) supported on silica wool, and pure silica wool (c).

The characteristic peaks of dioxymethylene species located at 1128 and $968 \mathrm{~cm}^{-1}$ and monodentate formate species at $1303 \mathrm{~cm}^{-1}$ emerged after exposure of $\mathrm{O}_{2}+\mathrm{HCHO}+\mathrm{H}_{2} \mathrm{O}+\mathrm{N}_{2}$ gas mixture over Co@NC-1.0 and Co@NC-0.5 (Figure S16a and b), being similar to those of Co@NC-0.25, and implying the catalytic transformation of HCHO. The signals appeared at the range of $3850 \sim 3610 \mathrm{~cm}^{-1}$ and $1700 \sim 1500 \mathrm{~cm}^{-1}$ might originate from the moisture and very small amount of $\mathrm{HCOOH}$ in the reactant gas, and were also observed over pure silica wool (Figure S16c). 


\section{REFERENCES}

(1) Luo, E.; Zhang, H.; Wang, X.; Gao, L.; Gong, L.; Zhao, T.; Jin, Z.; Ge, J.; Jiang, Z.; Liu, C.; Xing, W. Single-Atom Cr-N 4 Sites Designed for Durable Oxygen Reduction Catalysis in Acid Media. Angew. Chem. Int. Ed. 2019, 58 (36), 12469-12475.

(2) Kresse, G.; Furthmuller, J. Efficiency of ab-initio Total Energy Calculations for Metals and Semiconductors Using a Plane-Wave Basis Set. Comp. Mater. Sci. 1996, 6 (1), 15-50.

(3) Kresse, G.; Furthmuller, J. Efficient Iterative Schemes for ab initio Total-Energy Calculations Using a Plane-Wave Basis Set. Phys. Rev. B 1996, 54 (16), 11169-11186.

(4) Perdew, J. P.; Chevary, J. A.; Vosko, S. H.; Jackson, K. A.; Pederson, M. R.; Singh, D. J.; Fiolhais, C. Atoms, Molecules, Solids, and Surfaces-Applications of the Generalized Gradient Approximation for Exchange and Correlation. Phys. Rev. B 1992, 46 (11), 6671-6687.

(5) Macdonald, A. H. Comment on Special Points for Brillouin-Zone Integrations. Phys. Rev. $B$ 1978, 18 (10), 5897-5899. 